\title{
DIAGNOSTICO EM EDUCAÇÃO EM SAÚDE: UM MODELO PARA ANALISAR AS RELAÇÕES ENTRE ATITUDES E PRÁTICAS NA ÁREA DA SAÚDE PÜBLICA
}

Nelly Martins Ferreira Candeias* Ruth Sandoval Marcondes*

$\begin{array}{r}\text { RSPUB9/450 } \\ \hline\end{array}$

Candeias, N. M. F. \& MARCondes, R. S. Diagnóstico em educafão em saúde: ım modelo para analisar as relaçóes entre atitudes e práticas na área da saúde pública. Rev. Saúde públ., S. Paulo, 13:63-8, 1979.

RESUMO: São abordados aspectos relativos à natureza das inconsistências entre atitudes e práticas em termos de teorias psicossociais. Com vistas a facilitar a fase do diagnóstico no planejamento em educaf̧áo em saúde, apresenta-se um modelo para análise da situafão em que se encontra o objeto da saúde pública.

Unitermos: Educaşão em saúde pública, planejamento. Atitude em relação à saúde. CAP.

\section{TNTRODUCAO}

A afirmação de que a saúde física e mental é um dos valores mais significativos do ser humano é quase um lugar comum. Apesar disto, os indivíduos continuam a adotar práticas que, muito freqüentemente, acabam por afetar sua saude a curto, médio ou longo prazo. Às vezes, isto ocorre por ignorância; outras vezes, adotam-se determinadas práticas apesar de se ter consciência da potencialidade negativa de seus efeitos para a saúde. Ambos os fatos dizem respeito à educação em saúde.

Como parte do planejamento em educação em saúde cabe aos educadores fazer um diagnóstico dos conhecimentos (C), atitudes (A) e práticas (P) em saude do educando, ou seja, do CAP existente antes da intervenção educativa para, subseqüentemente, desenvolver atividades programadas que the permitam alcançar o CAP desejável do ponto de vista da saúde pública. Como afirma Derryberry 2, "educação em saúde diz respeito a um conjunto de experiências do individuo que modificam seus conhecimentos, atitudes e práticas, assim como o processo e esforços que visam a produzir tais modificações". Segundo Marcondes ", "educação em saúde é um processo essencialmente ativo que envolve uma mudança no modo de pensar, sentir e agir dos indivíduos, visando à obtenção da saúde, tal como definida pela OMS, ou seja, representando um bem-estar físico, mental e social. E, assim, o processo pelo cual os indivíduos mudam

* Do Departamento de Prática de Saúde Pública da Faculdade de Saúde Pública da USP Ar. Dr. Arnaldo, 715 - 01255 - São Paulo, SP - Brasil. 
CANDEIAS, N. M. F. \& MARCONDES, R. S. Diagnóstico em educação em saúde: um model para analisar as relações entre atitudes e práticas na área da saúde pública. Rev. Saúde públ., S. Paulo, 13:63-8, 1979.

ou adquirem conhecimentos, atitudes e práticas conducentes à saúde."

Tendo em vista este objetivo, surgiu na área da educação em saúde um interesse pelo estudo teórico e prático de modelos comportamentais, considerados como quadros analíticos para pesquisas em comunicação e programas de intervenção social. Simonds ${ }^{11}$ refere-se a um relatório da UNESCO, que enumera 25 modelos oriundos de diferentes disciplinas, tais como psicologia social, sociologia, psicologia, antropologia, economia, ciências biomédicas, demografia e comunicação. Segundo o autor, isto assume particular significado para educadores de saúde, porque amplia as perspectivas na fase do diagnóstico da situação na medida em que fornece opções teóricas que contribuem para compreensão do comportamento humano. Observa, entretanto, que quanto mais preciso for o modelo, mais reduzida será sua aplicação e menor acessibilidade a alternativas; ao contrário, quanto mais geral aquele for, menor será sua precisão e maior a acessibilidade a alternativas.

Dentre os modelos comportamentais experimentados, um deles tem-se mostrado demasiadamente simplista para ser utilizado na prática da educação em saúde: o processo conhecido pela designação CAP. Segundo este, um comportamento em saúde prende-se a um processo seqüencial: tem origem na aquisição de um conhecimento cientificamente correto, que pode explicar a formação de uma atitude favorável à adoção de uma determinada prática de saúde. De acordo com a premissa CAP, espera-se que um conhecimento cientificamente correto, na área da saúde, leve a uma mudança comportamental.

Este referencial teórico tem-se mostrado insuficiente para explicar os fenômenos relacionados à opção comportamental do ser humano no que respeita à manutenção de sua saúde. Ao analisar o conjunto de fatores que incidem sobre 0 ato de tomada de decisão, observa-se, muito freqüentemente, uma inconsistência entre conheci- mentos (o que se sabe), atitudes (o que se acha) e práticas (o que se faz) em relação ao objeto da saúde pública. Tal fenômeno ocorre mesmo entre os individuos mais racionais e informados a respeito de assuntos relacionados à medicina preventiva. Não é raro encontrar individuos que tiveram acesso a conhecimentos cientificamente corretos $(C+)$, acham que deveriam abandonar determinada prática $(\mathrm{A}+)$ e, não obstante, continuam a adotá-la (P-). O hábito de fumar ilustra, muitas vezes, uma situação do tipo $\mathrm{C}+\mathrm{A}+\mathrm{P}$ - Tal ocorrência torna-se mais compreensivel quando se tem em vista o fato de que as açōes derivam de um conjunto quase caótico de fatores e circunstâncias ambientais: apesar de o indivíduo valorizar a própriá saúde, outros valores mostram-se igualmente relevantes para a felicidade e bem-estar sociais. Sabe-se, há muito, que o homem não reage apenas em resposta a estímulos do meio ambiente, nem simplesmente em função de determinadas forças interiores, atuando em função do impacto concomitante dessas duas ordens de fatores ${ }^{11}$.

O estudo da inconsistência entre atitudes e práticas, na medida em que dificulta ou impede a consecução dos objetivos de programas de saúde pública, apresenta particular interesse para a educação em saúde. Torna-se oportuno, por isso, fazer breve revisão de algumas teorias de natureza psicossocial, que se propõem a explicar as características das relaçōes entre atitudes e práticas, tal como consideradas na premissa teórica CAP. Partindo do pressuposto de que os conceitos propostos por essas teorias facilitam o diagnóstico educativo e a intervenção social subseqüente, 0 presente trabalho pretende:

- fazer uma revisão de algumas teorias que visam a explicar o relacionamento entre atitudes e práticas, apresentando alguns exemplos concretos que explicam possiveis inconsistências na área do comportamento em saúde; 
CANDEIAS, N. M. F. \& MARCONDES, R. S. Diagnóstico em educação em saúde: um modelo para analisar as relações entre atitudes e práticas na área da saúde pública. Rev. Saúde públ., S. Paulo, 13:63-8, 1979.

- apresentar um modelo gráfico que permita aos responsáveis pelo planejamento em educação em saúde identificar e avaliar alguns fatores circunstanciais que podem interferir no processo de tomada de decisão do individuo, levando-o a aceitar ou rejeitar práticas desejáveis do ponto de vista da saúde pública.

\section{TEORIAS SOBRE RELACIONAMENTO} ENTRE ATITUDES E PRATICAS

\section{Enfoque da Consistência Cognitiva}

Denomina-se de "enfoque de consistência cognitiva", a afirmação teórica segundo a qual as atitudes dos individuos, medidas através de questionários mais ou menos sofisticados, representam indicadores válidos do comportamento humano. Partindo-se de tal pressuposto, pode-se considerar uma atitude como percursora provável de um determinado padrão comportamental. Deste modo, se uma mulher expressa uma atitude favorável em relação ao pré-natal, pode-se esperar que compareça regularmente a serviços de atendimento pré-natal; se uma mulher demonstra uma atitude favorável a exames de prevenção de câncer ginecológico, pode-se supor, igualmente, que a mesma se submeta periodicamente a um exame dessa natureza.

Este enfoque tem sofrido graves ataques dos pontos de vista metodológico e teórico ${ }^{7,9,11}$. Já em 1934, La Piere ${ }^{5}$ havia se referido ao problema decorrente de inconsistências entre atitudes e práticas. Subseqüentemente, outros autores chamaram a atenção para esse mesmo aspecto. Estudos realizados na área do comportamento preventivo, por Hassinger e McNamara 4, e da administração, por Deutscher ${ }^{3}$, confirmaram resultados apresentados por La Piere 5. Em 1940, o sociólogo Mills ${ }^{8}$ apontou a disparidade entre o que se diz e o que se faz como um problema metodológico central na área das ciências sociais.
O enfoque de consistência cognitiva, com seus pressupostos da correspondência esperada entre atitudes e práticas, foi impugnado não apenas do ponto de vista empírico, mas também do ponto de vista teórico. A crítica mais representativa surgiu dos interacionistas simbólicos. Para Blumer ${ }^{1}$, a disposição de predizer o comportamento humano a partir de atitudes em relação a dado objeto reduz o ser humano a um organismo passivo.

O conjunto de críticas sistemáticas ao enfoque de consistência cognitiva mostrou que, em saúde pública, não basta apenas conhecer as atitudes do individuo em relação a determinado objeto. Torna-se necessário, também, analisar as atitudes no que respeita à situação em que aquele objeto se encontra. Tal constatação levou ao desenvolvimento do enfoque da consistência contigente, cujos representantes mais significativos se apresentam a seguir.

\section{Enfoque da Consistência Contingente}

O enfoque da consistência contingente interpreta consistências entre atitudes e práticas, não meramente a partir da atitude do educando em relação ao objeto ( $\mathrm{AO}$ ), como na abordagem anterior, mas, também, a partir da sua atitude em relação à situação em que o mesmo se encontra (AS). Segundo Rokeach 10, o comportamento ou as práticas dos individuos decorrem de uma atitude em relação a dado objeto e de uma atitude em relação às circunstâncias que envolvem esse objeto. Estas circunștâncias podem prender-se a normas sociais, valores culturais e papéis, entre outros fatores ${ }^{12}$. Portanto, a partir desta premissa teórica, para entender o comportamento humano torna-se necessário analisar duas ordens de atitudes $(C=A O$ + AS).

Para fins de complementação, cumpre mencionar alguns exemplos desse enfoque na área da saúde pública. Uma gestante pode apresentar uma atitude positiva em relação ao comparecimento à assistência 
CANDEIAS, N. M. F. \& MARCONDES, R. S. Diagnóstico em educação em saúde: um modelis para analisar as relações entre atitudes e práticas na área da saúde pública. Rev. Saúde públ., s. Paulo, 13:63-8, 1979.

pré-natal $(\mathrm{AO}+)$ e ter, por outro lacio, uma atitude negativa ( $\mathrm{AS}-$ ) em relação à situação ou às circunstâncias que envolvem essa assistència: 0 fato de 0 centro de saúde ser distante de sua casa, de o tempo de espera ser muito longo, de alguns elementos da equipe de saúde não a atenderem adequadamente, ou de atemorizar-se com o exame ginecológico podem interferir negativamente em sua decisão. Outro exemplo: um individuo pode apresentar uma atitude positiva em relação à necessidade de deixar de fumar ( $\mathrm{AO}+$ ), mas reconhecer por outro lado, que o fumo o tranquiliza (AS-), permitindo-lhe melhor controle das situações problemáticas que provocam 0 aumento de tensões emocionais.

Uma outra versão teórica do enfoque de consistência contingente foi apresentada por Yinger ${ }^{13}$ para quem, em termos de teoria de campo, deve-se considerar a totalidade dos fatos coexistentes, assim como sua interdependência mútua. Refere-se o autor a "apoios estruturais" e ao seu impacto sobre o comportamento do individuo. Para Yinger ${ }^{13}$, é preciso considerar dois tipos de individuos: os individuos que mantêm uma consistência permanente entre atitudes e práticas, mesmo na ausência de "apoios estruturais", e os individuos ambivalentes, yue alteram seu comportamento em função de pressões momentâneas. Afirma-se, assim, que 0 individuo pode modificar seu comportamento na presença ou ausência de determinados "apoios estruturais" ou reforços. Um exemplo prático permite entender melhor a argumentação deste autor: um individuo obeso pode mostrar-se interessado em adotar a dieta recomendada pelo médico durante uma consulta, abandonando tal idéia ao regressar à sua casa, onde inexistem apoios estruturais. Neste caso, caberia aos responsáveis pelo processo educativo, proporcionar uma atmosfera favorável capaz de intensificar e criar novos apoios estruturais como, por exemplo, discussões de grupo, utilização de recursos audiovisuais e educação familiar. entre outros.
Para analisar a consistência entre atitudes e práticas deve-se considerar, também, um outro fator interacional, a que Warren e De Fleur"2 denominam de "coerção social". As situações de alta coerção social ocorrem sempre que 0 individuo interage com seu grupo de referência, permitindo que o mesmo tome conhecimento imediato de seu comportamento. Ao contrário, em situações de baixa coerção social, o anonimato individual encontra-se protegido de modo que a opinião e a ação do indivíduo não se sujeitam ao escrutínio alheio. Neste contexto, pode interpretar-se o medo à crítica como uma forma de alta pressão social. O exemplo a seguir ilustra esta situação: um adolescente pode inclinar-se a largar o fumo por ter tido informações a respeito de seus efeitos sobre a saúde, continuando, porém, a fumar porque os companheiros tambén fumam e teme perder o prestígio junto ao seu grupo de referência positiva por abandonar uma prática unanimemente aceita.

Em resumo, do ponto de vista do enfoque de consistência contingente, para que não haja inconsistência entre atitudes e práticas é preciso considerar, de modo geral, a situação em que o objeto se encontra ${ }^{10}$. Os conceitos de "apoios estruturais" e de "coerção social" devem ser utilizados no diagnóstico da situação em que o objeto se encontra 12,13 .

DIAGNÓSTICO EM EDUCAÇAO EM SAODE: UM MODELO PARA ANALISE DAS RELAÇOES ENTRE ATITUDES E PRATICAS NA AREA DA SAÚdE PÚBLICA

A partir destas considerações de caráter teórico, e tendo em vista a fase de diagnóstico em educação em saúde, elaborou-se um modelo para análise da situação em que se encontra o objeto de interesse para a saúde priblica (Vide Figura). 
CANDEIAS, N. M. F. \& MARCONDES, R, S. Diagnóstico em educação em saúde: um models para analisar as relações entre atitudes e práticas na área da saúde pública. Rev. Saúde públ., S. Paulo, 13:63-8, 1979.

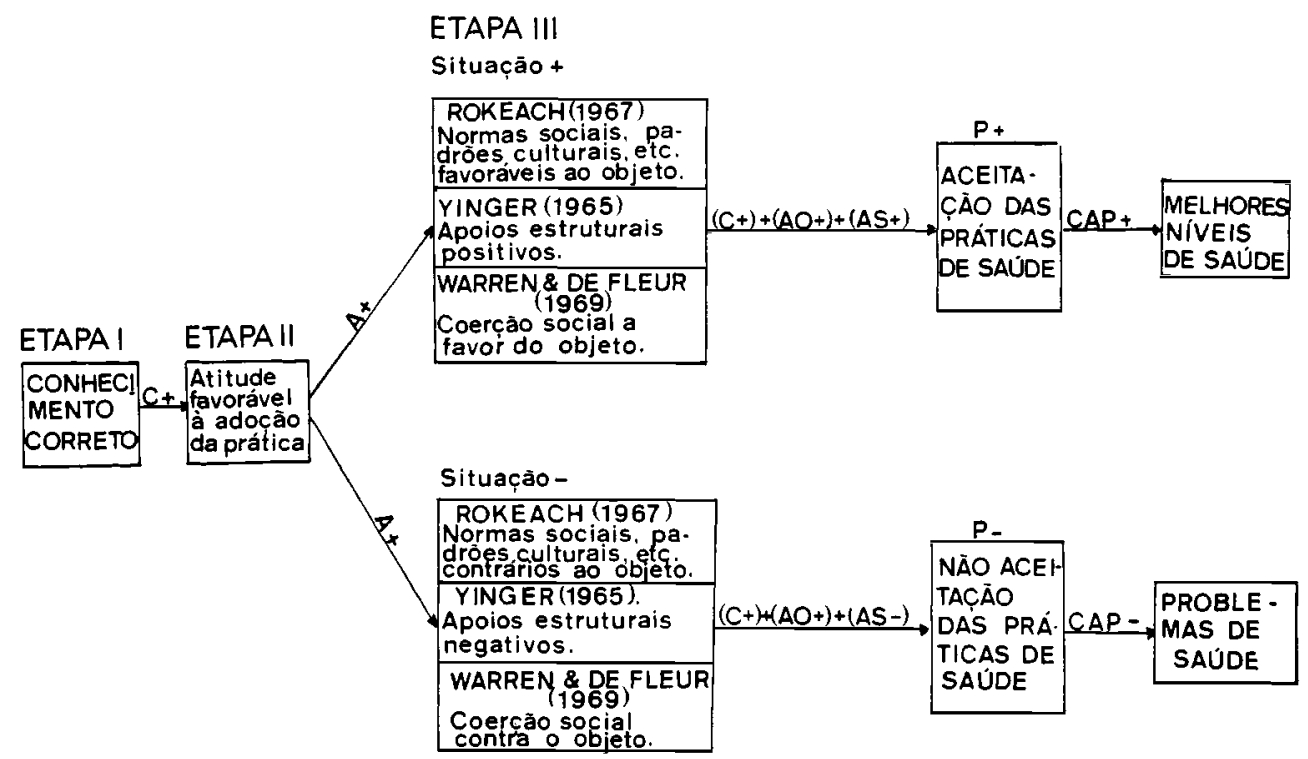

Diagnóstico em Educação em Saúde Pública - Modelo para análise das relaçōes entre atitudes e práticas na área da saúde pública.

Cumo se observa na Fig., o educando pode ter sido exposto a um conhecimento cientificamente correto sobre determinado objeto de saúde $(C+)$, apresentar uma atitude favorável em relação a este $(\mathrm{A}+)$ e encontrar, no ambiente médico, fatores situacionais positivos $(\mathrm{S}+)$. Neste caso, passa a adotar a prática recomendada, adquirindo assim um CAP positivo (CAP + ) que the permite alcançar melhores niveis de saude.

Pode ocorrer, também, que o educando adquira conhecimentos cientificamente corretos em relação a determinado objeto, apresente uma atitude favorável quanto a este, sem alcançar um CAP positivo $(\mathrm{CAP}+)$ em decorrência da situação negativa ( $\mathrm{S}-$ ) do ambiente em que o mesmo se encontra. O CAP negativo (CAP-) será responsável, nestas circunstâncias, por determinados problemas de saúde do individuo, que poderiam ser controlados ou eliminados a partir da perspectiva da medicina preventiva.

\section{CONSIDERAÇOES FINAIS}

De acordo com os estudos de Rokeach ${ }^{10}$, Yinger ${ }^{13}$, Warren e De Fleur ${ }^{12}$ é preciso considerar as atitudes dos educandos não só em relação a determinado objeto, mas também em relação à situação em que o mesmo se encontra (AO $+A S)$.

No processo de planejamento em educação em saúde pública, tendo em vista a fase de diagnóstico, cabe aos educadores fazer um levantamento dos conhecimentos da população-alvo em relação a determinada ação social e controlar, na medida do possivel, as atitudes em relação ao objeto e, parelelamente, às circunstâncias situacionais que o rodeiam (AO $+\mathrm{AS}$ ). Só a partir do controle de fatores circunstanciais negativos, alguns dos quais se apresentam no presente trabalho, pode o educando alcançar um CAP positivo (CAPH) que the garanta, dentro de uma perspectiva preventiva, melhores niveis de saúde. 
CANDEIAS, N. M. F. \& MARCONDES, R. S. Diagnóstico em educação em saúde: um modelo para analisar as relações entre atitudes e práticas na área da saúde pública. Rev. Saúde puibl., S. Paulo, 13:63-8, 1979.

Candeias, N. M. F. \& Marcondes, R. S. /Diagnosis in health education: a model for analysis of relationships between attitudes and practices in the area of public health]. Rev. Saúde públ., S. Paltlo, 13:63-8, 1979.

ABSTRACT: The nature of inconsistencies between attitudes and practices is examined in terms of social-psychological theories. To facilitate diagnosis in health education, a model for the analysis of situational factors which affect a person's behavior is presented.

UNITERMS: Health education, planning. Attitude to health. KAP.

\section{REFERENCIAS BIBLIOGRAFICAS}

1. BLUMER, H. Sociological implications of the thought of G. H. Mead. Amer. Sociol., $71: 535,1966$.

2. DERRYBERRY, M. Health education: its objectives and methods. Fith Educ. Monogr., 8:3-9, 1960.

3. DEUTSCHER, I. Words and deeds: social science and social policy. Soc. Probl., $13: 235-54$, 1966.

4. HASSINGER, E. \& MCNAMARA, R. L. Stated opinions and actual practice in health behavior in a rural area. Midwest. sociol., 4:93-7. 1957.

5. LA PIERE, R. T. Attitudes vs. action. Soc. Forces, 43:230-7, 1984.

6. MARCONDES, R. S. Educação em saude pública: conceituacão, objetivos $e$ principios. São Paulo, Faculdade de Saúde Pública USP. Disciplina Educação em Saúde Pública, 1974. [mimeografado].

7. McGUIRE, W. J. The current status of cognitive consistency theories. In: Fishbein, M., ed. Readings in attitude theory and measurement. New York, John Wiley and Sons, 1967, p. 401-422.
8. MILLS, C. W. Methodological consequences of the sociology of knowledge. Amer. J. Sociol,, 46:316-30, 1946.

9. RICHARDS, N. D. Methods and effectiveness of health education: the past. present and future of social scientific involvement. Soc. Sci. \& Med, 9: 141-56. 1975 .

10. ROKEACH, M. Attitude change and behavioral change. Publ. Opinion Quart.. $30: 529-50,1967$.

11. SIMONDS, S. K. Emerging challenges in health education. Int. J. Hlth Educ, 19(suppl. 40), 1976.

12. WARREN, L. G. \& DE FLEUR, M. L Attitude as an interactional concept: social constraint and social distance as intervening variables between attitudes and action. Amer. sociol. Rev., 34:153-69, 1969.

13. YINGER, J. M. Toward a field theory of behavior. New York, McGraw-Hill, 1965.

Recebido para publicação em 13/10/1978

Aprovado vara publicacão em 22/03/1979 